\title{
Peripheral ENO1-specific T cells mirror the intratumoral immune response and their presence is a potential prognostic factor for pancreatic adenocarcinoma
}

\author{
ELENA NICCOLAI $^{*}$, PAOLA CAPPELLO ${ }^{2 *}$, ANTONIO TADDEI $^{3}$, FEDERICA RICCI $^{1}$, MARIO MILCO D'ELIOS $^{1}$, \\ MARISA BENAGIANO ${ }^{1}$, PAOLO BECHI ${ }^{3}$, LAPO BENCINI ${ }^{4}$, MARIA NOVELLA RINGRESSI ${ }^{3}$, \\ ANDREA CORATTI $^{4}$, FABIO CIANCHI ${ }^{3}$, LISA BONELLO ${ }^{2,5}$, PAOLA FRANCIA DI CELLE ${ }^{5}$, \\ DOMENICO PRISCO ${ }^{1}$, FRANCESCO NOVELLI ${ }^{2}$ and AMEDEO AMEDEI ${ }^{1}$
}

\begin{abstract}
${ }^{1}$ Department of Experimental and Clinical Medicine, University of Florence, and Department of Biomedicine, Azienda Ospedaliera Universitaria Careggi (AOUC), I-50134 Florence; ${ }^{2}$ Centre for Experimental Research and Medical Studies (CERMS), AOU City of Health and Science of Turin, and Department of Molecular Biotechnology and Health Sciences, University of Turin, I-10126 Turin; ${ }^{3}$ Department of Surgery and Translational Medicine, University of Florence, and Department of Biomedicine, Azienda Ospedaliera Universitaria Careggi (AOUC), I-50134 Florence; ${ }^{4}$ Division of General and Oncologic Surgery, Department of Oncology, Azienda Ospedaliera Universitaria Careggi (AOUC), I-50134 Florence; ${ }^{5}$ General Anatomopathology and Molecular Oncogenetics - AOU City of Health and Science of Turin, I-10126 Turin, Italy
\end{abstract}

Received January 22, 2016; Accepted February 29, 2016

DOI: 10.3892/ijo.2016.3524

\begin{abstract}
Pancreatic ductal adenocarcinoma (PDAC) is an aggressive disease with an average survival of 4-6 months following diagnosis. Surgical resection is the only treatment with curative intent, but resectable PDAC patients are in the minority. Also, unlike other neoplasms, PDAC is resistant to conventional and targeted chemotherapy. Innovative treatments, such as immunotherapy, can be very important and the study of the immune response is fundamental. We previously demonstrated that PDAC patients show tumor-infiltrating T cells specific to $\alpha$-enolase (ENO1), a glycolytic enzyme overexpressed by pancreatic tumor cells, which plays an important role in promoting cell migration and cancer metastasis. In the present study, we evaluate the functional anticancer proprieties of ENO1-specific T cells isolated from the peripheral blood of
\end{abstract}

Correspondence to: Professor Amedeo Amedei, Department of Experimental and Clinical Medicine, Section of Internal Medicine University of Florence, Largo Brambilla 3, I-50134 Florence, Italy E-mail: aamedei@unifi.it

*Contributed equally

Abbreviations: ENO1, $\alpha$-enolase; MDSC, myeloid-derived suppressor cells; PDAC, pancreatic ductal adenocarcinoma; TCR, T cell receptor; Th, T helper; TILs, tumor-infiltrating lymphocytes; Tregs, regulatory $\mathrm{T}$ cells

Key words: pancreatic ductal adenocarcinoma, $\alpha$-enolase, $\mathrm{T}$ cells, prognosis, $\mathrm{T}$ cell receptor repertoire
PDAC patients. Furthermore, comparing the T cell receptor repertoire of ENO1-specific peripheral and infiltrating tumor $\mathrm{T}$ cells from the same patient suggests that ENO1-specific $\mathrm{T}$ cells, despite having a different functional profile, can recirculate from the tumor to the periphery. Finally, of clinical relevance, the presence of peripheral ENO1-specific $\mathrm{T}$ cells has a prognostic value and significantly correlates with a longer survival.

\section{Introduction}

Patients affected by PDAC have an extremely poor prognosis, with a 5-year survival rate of only $\sim 4-5 \%$ and a mean survival of 4-6 months. Surgical resection is the only treatment with curative intent, but patients with resectable PDAC are in the minority (1). Upon tumor resection, the 5-year survival rate increases to approximately $15 \%$, while $25 \%$ survival is attained from treatment with adjuvant chemotherapy (2). There are many reasons for such a poor prognosis, including rapid tumor dissemination and late diagnosis (3), due to the lack of early, disease-specific symptoms. One of the most important features of PDAC is the early occurrence of immune suppression that affects the $\mathrm{T}$ cell-mediated antitumor response. In general, immune suppression is manifested in two different ways: firstly, by immune suppressive cells, such as regulatory $\mathrm{T}$ cells (Tregs) and myeloid-derived suppressor cells (MDSC) and secondly, by surface regulatory molecules, such as CTLA-4 and PD-L1. In human PDAC, B7-H1 expression was upregulated and correlated strongly with poor patient prognosis (4) while a decrease in the number of $\mathrm{CD} 4^{+} \mathrm{T}$ cells expressing CTLA-4 is associated with improved and disease-free survival of PDAC patients (5). 
However, different studies report an antitumor immune response in PDAC patients, for example, accumulation of $\mathrm{CD}^{+} \mathrm{T}$ cells correlates with increased survival of patients (5-7), supporting the protective role of the immune system against developing tumors. In fact, usually, the PD-L1 expression has a significant inverse correlation with $\mathrm{CD}^{+}{ }^{+}$TILs and CD8 ${ }^{+}$TILs $(8,9)$.

In cancer immunology, the balance of co-stimulatory or inhibitory responses is an important underlying mechanism in the function of $\mathrm{T}$ cell-mediated anticancer immunity.

In particular, the PD-1/PD-L1 axis is one of the prominent immune checkpoints, which are known as the inhibitory mechanisms of immune responses. The linkage of PD-L1 of the tumor cells with PD-1 expressed on the activated tumorinfiltrating lymphocytes (TILs), induces a co-inhibitory signal and decreases the level of cytokines, which subsequently results in the anergy and apoptosis of the effector T lymphocytes and to a poor patient prognosis (10). Thus, although $\mathrm{CD}^{+} \mathrm{T}$ cells were thought to be the effective antitumor T-cell subset, some studies did not find any correlation between $\mathrm{CD}^{+} \mathrm{T}$-cells and survival rate in patients with pancreatic cancer (11).

The immune response elicited by the deregulated expression of various tumor-associated antigens (TAA) has been demonstrated in different cancers $(12,13)$, including PDAC, as shown by serological profiling that identified 29 preferentially immunogenic proteins in the serum of PDAC patients $(14,15)$. Numerous data have demonstrated that PDAC patients generate T/B cells against TAA (16-22), including $\alpha$-enolase (ENO1) which we have previously described $(23,24)$ and that patients with anti-TAA antibodies had a better survival rate than the antibody-negative patients $(25,26)$. Vaccination against ENO1, a glycolytic enzyme that plays a role in cell migration and cancer metastasis (27), delays tumor progression and significantly extends survival in a mouse model of PDAC (28). As ENO1 can be considered as a promising candidate for immunotherapy in PDAC, information on the antitumor function of ENO1-specific T cells from peripheral blood lymphocytes may provide a prognostic tool.

Naive $\mathrm{CD}^{+} \mathrm{T}$ cells differentiate into mature Th1, Th2, Th17 or Tregs. Th1 cells play an effective anticancer role, while Th2, and especially Tregs, suppress immune responses and induce tolerance at tumor sites, particularly in $\operatorname{PDAC}(29,30)$. There are conflicting reports, however, on the role of Th17 cells in cancer (31), with some studies reporting that higher levels of Th17 cells correlate with advanced cancer $(32,33)$, while others suggest that Th17 may have a potent antitumor effect (34).

The aim of the present study was to characterize the phenotypic and functional properties of ENO1-specific T-cells isolated from the blood of PDAC patients, evaluating their anticancer functional role and to ascertain whether they are able to recirculate from the tumor to the periphery and vice versa. Finally, we correlated the presence of peripheral antiENO1 T cells with patient survival.

\section{Materials and methods}

Patients. For the present study, we used PBMCs (peripheral blood mononuclear cells) from 15 patients ( 8 males and 7 females) with pancreatic ductal adenocarcinoma (according to the TNM classification for pancreatic tumors) (35), who were already enrolled in a previous study approved by the local Ethics Committee (24). The age of PDAC patients ranged from 36 to 92 years (mean age, 63 years). All patients underwent surgical resection of the primary lesion but did not receive chemotherapy. Patients with evidence of serious illness, immunosuppression, autoimmune or infectious diseases were excluded. Characteristics of PDAC patients and ENO1 expression of PDAC tissue are summarized in Table I. For comparison with healthy controls, 15 blood donors (8 males and 7 females, mean age 60) were studied.

Reagents. T cell cultures were carried out in RPMI-1640 culture medium (SERO-Med GmbH, Vienna, Austria) supplemented with 10\% FCS HyClone Laboratories (Gibco Laboratories, Grand Island, NY, USA) and recombinant human interleukin-2 (IL-2) (Eurocetus, Milan, Italy). Unlabeled or fluorochromeconjugated anti-CD3, CD4, CD8, CD25, and isotype-matched control mAbs were purchased from BD Biosciences (San Jose, CA, USA). Fluorochrome-conjugated anti-IL-10, anti-TGF- $\beta$ and anti-FoxP3 mAbs were purchased from eBioscience (San Diego, CA, USA).

Production of recombinant histidine-tagged a-enolase and detection of serum anti-ENO1 immunoglobulin $G(\operatorname{IgG})$. Recombinant $\alpha$-enolase was overexpressed in the $E$. Coli strain BL21 (DE3)/pLysS (kindly provided by A. Giallongo, Institute of Biomedicine and Molecular Immunology, National Research Council, Palermo, Italy) and was produced as previously reported (23). Anti-ENO1 IgG levels were measured by an in-house ELISA kit (23).

Detection of CA19-9 marker. The fasting venous blood $(2 \mathrm{ml})$ at the early morning of the study objects was drawn and the serum was centrifuged and stored at $-20^{\circ} \mathrm{C}$ until assayed. The electrochemiluminescence immunoassay method was used for detecting the levels of CA19-9. The procedures were conducted strictly according to the instructions of kits. The critical value of CA19-9 was $37 \mathrm{U} / \mathrm{ml}$.

Immunohistochemical analysis of ENO1. Immunohistochemical analysis on 15 PDAC tissues was performed with an anti- $\alpha$-enolase $\mathrm{mAb}(1: 100$; clone 19/12) followed by a horseradish peroxidase (HRP)-conjugated goat anti-mouse IgG (Santa Cruz Biotechnology, Celbio s.p.a., Milan, Italy) and DAB detection kit (Ventana Medical Systems, Inc., Tucson, AZ, USA). Samples were fixed with formalin and paraffin-embedded. Slides were incubated with the primary and secondary $\mathrm{Ab}$ and developed in a Ventana ES automated stainer. Stained sections were counterstained with hematoxylin.

All images were taken with a x60 objective. Images were acquired on a Leica DM LA upright microscope equipped with a DC300F camera and were analyzed with IM50 software (Leica Microsystems, Heidelberg, Germany).

Detection of ENO1-specific T cells in the peripheral blood of patients with PDAC. To assess the presence of ENO1-specific $\mathrm{T}$ cells in the peripheral blood of PDAC patients, PBMCs obtained by density gradient centrifugation on Ficoll-Hypaque were re-suspended in medium supplemented with $3 \%$ human 
Table I. Patient characteristics and ENO1-reactivity of T cell clones (Tcc) generated from blood of patients with pancreatic ductal adenocarcinoma.

\begin{tabular}{lclcccccc}
\hline & $\begin{array}{c}\text { Overall } \\
\text { survival } \\
\text { Code }\end{array}$ & $\begin{array}{c}\text { Tumor } \\
\text { grade }\end{array}$ & $\begin{array}{c}\text { CA19-9 } \\
\text { (U/ml) }\end{array}$ & $\begin{array}{c}\text { ENO1 } \\
\text { expression }\end{array}$ & $\begin{array}{c}\text { Anti-ENO1 } \\
\text { IgG }\end{array}$ & $\begin{array}{c}\text { ENO1-PBMC } \\
\text { proliferation }\end{array}$ & Peripheral Tcc & $\begin{array}{c}\text { Peripheral ENO1- } \\
\text { specific Tcc (\%) }\end{array}$ \\
\hline 01.PCp & 11 & Well & $400 \pm 21$ & + & ++ & $\mathrm{Y}$ & 16 & $4(25)$ \\
02.PCp & 13 & Moderate & $420 \pm 28$ & ++ & ++ & $\mathrm{Y}$ & 20 & $5(25)$ \\
03.PCp & 8 & Poor & $570 \pm 35$ & + & + & $\mathrm{N}$ & 0 & - \\
04.PCp & 7 & Well & $850 \pm 62$ & +++ & + & $\mathrm{N}$ & 10 & $2(20)$ \\
05.PCp & 9 & Well & $1100 \pm 42$ & +++ & + & $\mathrm{Y}$ & 15 & $3(20)$ \\
06.PCp & 8 & Moderate & $1250 \pm 31$ & ++ & + & $\mathrm{N}$ & 0 & - \\
07.PCp & 12 & Well & $210 \pm 15$ & ++ & ++ & $\mathrm{Y}$ & 15 & $5(33)$ \\
08.PCp & 8 & Moderate & $830 \pm 68$ & +++ & + & $\mathrm{Y}$ & 10 & $2(20)$ \\
09.PCp & 14 & Poor & $150 \pm 45$ & +++ & +++ & $\mathrm{Y}$ & 20 & $8(40)$ \\
10.PCp & 9 & Poor & $1350 \pm 89$ & + & - & $\mathrm{N}$ & 0 & - \\
11.PCp & 10 & Well & $135 \pm 24$ & + & ++ & $\mathrm{Y}$ & 15 & $6(40)$ \\
12.PCp & 11 & Poor & $216 \pm 21$ & ++ & ++ & $\mathrm{Y}$ & 14 & $4(29)$ \\
13.PCp & 8 & Well & $897 \pm 57$ & +++ & + & $\mathrm{N}$ & 10 & $2(20)$ \\
14.PCp & 12 & Moderate & $235 \pm 18$ & + & ++ & $\mathrm{Y}$ & 12 & $4(33)$ \\
15.PCp & 13 & Well & $326 \pm 35$ & +++ & +++ & $\mathrm{Y}$ & 24 & $9(38)$ \\
All PDAC & 10.2 & Well & $384.6 \pm 39$ & $15 / 15$ & $14 / 15$ & $10 / 15$ & 181 & 54 \\
patients & & $(47 \%)$ & & & $(93 \%)$ & $(67 \%)$ & & $(30 \%)$ \\
\hline
\end{tabular}

PBMC, peripheral blood mononuclear cells; Y, yes, N, no. To rank the presence of anti-ENO1-IgG we referred to a threshold of the OD value $+3 \mathrm{SD}$ from 45 age-matched sera from healthy subjects $(0.13)$. The range between 0.13 and the higher value from PC patient sera $(0.622)$ was divided in 3 and assigned $(+)$ for OD values between 0.13 and $0.294,(++)$ for OD between 0.294 and 0.458 , and (+++) for OD between 0.458 and 0.622 .

serum. PBMCs $\left(3 \times 10^{5}\right)$ were cultured for $96 \mathrm{~h}$ in the presence of medium alone or ENO1 $(10 \mu \mathrm{g} / \mathrm{ml})$. At $16 \mathrm{~h}$ before harvesting, $0.5 \mu \mathrm{Ci} /$ well $\left[{ }^{3} \mathrm{H}\right]$-thymidine (Amersham Pharmacia Biotech, Uppsala, Sweden) was added, and the radionuclide uptake was measured by a $\beta$-counter. The mitogenic index (MI) was calculated as the ratio between mean values of cpm (counts per minute) obtained in ENO1-stimulated cultures and those obtained in the presence of medium alone. An MI $\geq 3$ was considered as a positive result.

Generation of ENO1-specific $T$ cell lines and $T$ cell clones (Tcc). ENO1-specific T cell lines were generated as previously described (25). Briefly, $10^{6}$ PBMCs in $2 \mathrm{ml}$ RPMI-1640 medium supplemented with $2 \mathrm{mM}$ L-glutamine, $2 \times 10^{-5} \mathrm{M}$ 2-ME, and 5\% human serum (complete medium) were stimulated with ENO1 $(10 \mathrm{mg} / \mathrm{ml})$ in 24 -well flat-bottomed plates for 5 days. Then, human IL-2 (30 U/ml) was added, and cultures were maintained for 7 days. Viable T-cell blasts were re-suspended in complete medium and cloned under limiting dilution, as previously described (36). Briefly, single T cell blasts were seeded in microwell plates $(0.3$ cells/well $)$ in the presence of $2 \times 10^{5}$ irradiated (9000 rad) PBMCs, phytohemagglutinin (PHA) $(0.5 \% \mathrm{vol} / \mathrm{vol})$ and IL-2 (50 U/ml). At weekly intervals, $2 \times 10^{5}$ irradiated PBMC and IL-2 were added to each microculture to maintain the expansion of growing clones.
Tcc were screened for responsiveness to ENO1 by measuring $\left[{ }^{3} \mathrm{H}\right]$-thymidine uptake after a 60 -h co-culture with irradiated autologous mononuclear cells in the presence of medium or ENO1 $(10 \mathrm{mg} / \mathrm{ml})$. An MI $\geq 3$ was considered as a positive result.

To verify the clonality of ENO1-specific Tcc, the repertoire of the T cell receptor (TCR) V $\beta$ chain of single Tcc was analyzed with the TCR V $\beta$ Repertoire kit (Beckman Coulter, Fullerton, CA, USA); isotype-matched non-specific IgG were used as negative controls and the $\mathrm{T}$ cell blasts were gated by anti-CD3 monoclonal antibody.

Characterization of the cytokine profile of Tcc and analysis of surface/intracytoplasmic cell markers. We analyzed surface marker (CD3, CD4 and CD8) expression in blasts of single Tcc by flow cytometry as previously described (37), along with the production of the intracellular marker (FoxP3) and cytokines (IL-10 and TGF- $\beta$ ) of ENO1-specific Tcc (unable to secrete IFN- $\gamma$, IL-4 and IL-17). For intracellular analysis, blasts were stimulated with PMA (phorbol myristate acetate) (10 ng/ml) plus ionomycin $(200 \mathrm{ng} / \mathrm{ml})$, and stained with anti-IL-10, anti-TGF- $\beta$ and anti-FoxP3 mAbs. Tcc that were negative for FoxP3, IL-10 and TGF- $\beta$ were defined as T null, and Tcc that were positive for FoxP3, IL-10 and TGF- $\beta$ were defined as Tregs. 
To evaluate the amount of secreted cytokines, $\mathrm{T}$ cell blasts of single Tcc were resuspended at a concentration of $10^{6}$ cells $/ \mathrm{ml}$ of medium and cultured for $36 \mathrm{~h}$ in the presence of PMA (10 ng/ml) plus ionomycin (200 ng/ml). Cell-free supernatants were collected and assayed in duplicate for IFN- $\gamma$, IL-4 and IL-17 content by commercial ELISA kits (BioSource International, Camarillo, CA, USA). Supernatants presenting cytokine levels that were $5 \mathrm{SD}$ above the mean levels in control supernatants derived from irradiated APCs alone were considered positive. Based on the cytokine profile and the CD4/CD8 expression evaluation, we divided the clones into the following groups: Th1/Tc1 (only IFN- $\gamma$ ), Th2/Tc2 (only IL-4), Th0/Tc0 (IFN- $\gamma$ and IL-4) and Th17/Tc17 (IL-17).

$B-E B V$ cell preparation. B cells were prepared using the B cell isolation kit II (Miltenyi Biotec GmbH, Bergisch Gladbach, Germany). To obtain EBV-transformed lymphoblastoid B cell lines (EBV-B cells), autologous B cells from each patient were incubated for $48 \mathrm{~h}$ with EBV-producing marmoset B95.8 cell line supernatants, and subsequently expanded in complete medium supplemented with $15 \%$ FCS.

Perforin-mediated cytotoxicity and Fas-Fas ligand (L)-mediated proapoptotic activity. Perforin-mediated cytolytic activity of Tcc was assessed as previously reported (24). $\mathrm{T}$ cell blasts of ENO1-specific T clones were incubated at an effector-to-target ratio of 10-, 5- and 2.5 to-1 with ${ }^{51} \mathrm{Cr}$-labeled autologous (EBV)-B cells pre-incubated with ENO1 $(1 \mu \mathrm{g} / \mathrm{ml})$. After centrifugation, microplates were incubated for $8 \mathrm{~h}$ at $37^{\circ} \mathrm{C}$, and $0.1 \mathrm{ml}$ supernatant was removed from each microculture for measuring ${ }^{51} \mathrm{Cr}$ release. To evaluate whether Tcc-specific cytotoxicity against target cells was ENO1/HLA-dependent, cytotoxicity tests were also carried out under two other different conditions: i) with ENO1-pulsed ${ }^{51} \mathrm{Cr}$-labeled autologous EBV-B cells in the presence of anti-HLA-DR blocking $\mathrm{mAbs}(10 \mathrm{mg} / \mathrm{ml})$ to evaluate the ENO1-specific $\mathrm{CD}^{+} \mathrm{Tcc}$; ii) in the presence of anti-HLA-A-B-C to assess the ENO1specific $\mathrm{CD}^{+}{ }^{+}$Tcc. Anti-HLA-DR and anti-HLA-A-B-C mAbs were purchased from Immunotech (Beckman Coulter, Marseille, France).

The ability of ENO1-specific Tcc to induce Fas-FasLmediated apoptosis was assessed using $\mathrm{Fas}^{+} \mathrm{Panc1}$ cells as targets. T cell blasts from each clone were co-cultured with ${ }^{51} \mathrm{Cr}$-labeled Pancl cells at an effector-to-target ratio of 10-, 5and 2.5- to-1 for $18 \mathrm{~h}$ in the presence of PMA $(10 \mathrm{ng} / \mathrm{ml})$ and ionomycin $(200 \mathrm{ng} / \mathrm{ml})$, as previously reported (24). To block the Fas-FasL interaction, the anti-Fas antagonistic monoclonal antibody M3 (Amgen, Thousand Oaks, CA, USA) was used, at a final concentration of $5 \mathrm{mg} / \mathrm{ml}$, for a 30-min pre-treatment of ${ }^{51} \mathrm{Cr}$-labeled Pancl cells, as reported (24).

Suppressive assays. To assess the ability of antigen-specific Treg clones to suppress the antigen-induced autologous cell proliferation of ENO1-specific effector Tcc, $2 \times 10^{4}$ of these blasts were cultured with $4 \times 10^{3}$ irradiated (9000 rad) autologous ENO1-loaded or TT (Tetanus toxoid)-loaded dendritic cells (DCs) in the presence of $2 \times 10^{4}$ cells of ENO1-specific Treg. DCs were obtained using the Blood Dendritic Cell Isolation kit II (Miltenyi Biotec). At day 4 , after $8 \mathrm{~h}$ of pulsing with $0.5 \mathrm{mCi} 3 \mathrm{H}-\mathrm{TdR} /$ well (Amersham, Little Chalfont, UK), cultures were harvested and the radionuclide uptake was measured by $\beta$-counter analysis. Tetanus toxoid was used for specificity control of $\mathrm{T}$ cell clones.

DNA extraction and TCR Genescan analysis of TCR $\beta$ and $T C R \gamma$ rearrangement. Genomic DNA was extracted from ENO1-specific T cell clones obtained from three tumor samples and three peripheral blood samples of the same patient using the Maxwell 16 Tissue DNA Purification kit and the Maxwell 16 Instrument (Promega, Madison, WI, USA).

TCR gene rearrangement analysis was performed using a gene clonality assay based on the BIOMED-2/Euroclonality multiplex-PCR protocol (38), known as the IdentiClone TCR $\beta$ TCR $\gamma$ T-Cell Clonality Assa-ABI Fluorescence Detection (Invivoscribe, San Diego, CA, USA). The assay consists of five tubes each containing primers that target the TCR $\beta$ (tubes A-B-C) and the TCR $\gamma$ (tubes D-E) chain locus. Briefly, $100 \mathrm{ng}$ of genomic DNA per tube was subjected to PCR amplification according to the manufacturer's instructions. PCR products were run on an ABI 3130 Genetic Analyzer and Genescan profiles were obtained using GeneMapper (version 3.2) software (Applied Biosystems, Foster City, CA, USA).

Statistical analysis. Statistical analysis of the mitogenic index was evaluated by the unpaired two-tailed Student's t-test. A P-value $<0.05$ was considered statistically significant. A twotailed Pearson $r$ coefficient was used to correlate the titer of anti-ENO1 IgG and the percentage of ENO1-specific T clones obtained from PDAC patients. The correlation between the number of ENO1-specific Tcc and survival of patients and hazard ratio (HR) were evaluated by Kaplan-Meier analysis (log-rank Mentel-Cox test).

\section{Results}

ENO1-specific T cells in the peripheral blood of patients with $P D A C$. In order to assess the presence of ENO1-specific T cells in the peripheral blood of PDAC patients, PBMCs from 15 patients, which were previously characterized for the presence of tumor infiltrating T cells, (16) were cultured for 5 days in the presence of medium alone or recombinant ENO1. Notably, PBMCs from 10 of these PDAC patients (67\%) (Table I and Fig. 1A) proliferated in the presence of ENO1. Conversely, in the healthy controls, only 3 cases out of 15 (20\%) showed proliferation in response to ENO1. In addition, the proliferation of PBMC of PDAC patients showed a significantly greater mitogenic index (mean MI \pm SEM, 14.4 \pm 2.5 ) with respect to that of the three healthy controls (mean $\mathrm{MI} \pm \mathrm{SEM}, 3.3 \pm 0.8$; $\mathrm{P}=0.0002$ ).

Characterization of Tcc isolated from the blood of PDAC patients and reactivity to ENO1. As five PDAC patients did not have detectable ENO1-specific T cells in their peripheral blood, but four out of these five patients did, however, present ENO1-specific tumor-infiltrating T cells (16) we expanded and cloned peripheral blood lymphocytes, by IL-2, from all PDAC resectable patients; we obtained 181 Tcc from all PDAC patients except patients 3, 6 and 10 (Table I).

There were $88 \%(159 / 181)$ of $\mathrm{CD}^{+}$Tcc and $12 \%$ of $\mathrm{CD}^{+}$ Tcc (22/181). Interestingly, out of $22 \mathrm{CD}^{+} \mathrm{Tcc}, 7$ were isolated 

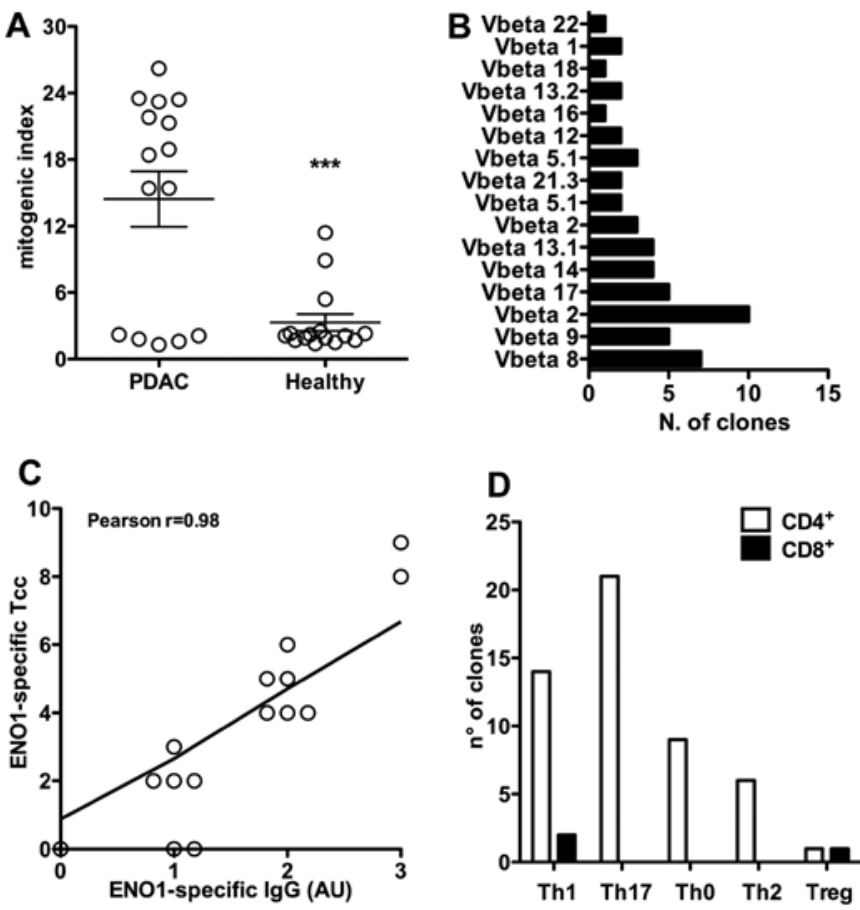

Figure 1. Detection of ENO1-specific T cells in the blood of PDAC patients and profile characterization of ENO1-specific Tcc. (A) The graph represents the mitogenic index of PBMC proliferation of ten PDAC patients and three healthy controls. PBMCs were stimulated with ENO1 and an $\mathrm{MI} \geq 3$ was considered as a positive result. PDAC patients showed a significantly greater mitogenic index $(\mathrm{P}=0.0002)$. (B) The histogram represents the TCR-V $\beta$ repertoire of the $54 \mathrm{CD} 4 / \mathrm{CD} 8 \mathrm{ENO} 1$-specific Tcc isolated from the blood of PDAC patients. (C) Correlation between the anti-ENO1 IgG titer and the number of ENO1-specific T clones obtained from the same PDAC patien (Spearman regression $\mathrm{r}=0.8745$ and $\mathrm{P}$-value $<0.0001$ ). (D) Cytokine profile distribution of ENO1-specific T cell clones.

from the blood of patient 5 Pcp, who was reported as having the highest number of $\mathrm{CD}^{+} \mathrm{Tcc}$.

As ENO1 is a TAA that induces an integrated humoral and cellular response in PDAC patients (15). Tcc obtained were assayed for proliferation in response to ENO1, and $30 \%$ of the Tcc was (54/181, Table I) proliferated upon stimulation with ENO1. With the exception of three cases (Pcp 3,6,10), we obtained ENO1-specific Tcc from all patients; in particular patients 9 and 15 were recorded as having the greatest number of Tcc (8 and 9, respectively).

Clonality was confirmed by cytofluorimetric analysis of TCR-V $\beta$ expression. Each Tcc was only revealed by one of the TCR-V $\beta$ chain-specific monoclonal antibodies, showing a single peak of fluorescence intensity (Fig. 1B).

We have already shown that all patients (except for patient PCp10) had detectable serum anti-ENO1 IgG (Table I). Interestingly, we observed that patients with higher titers of anti-ENO1 antibodies also showed the highest percentage of ENO1-specific T clones $(r=0.8745$ and $\mathrm{P}$-value $<0.0001)$ (Fig. 1C); particularly patients 9 and 15 .

Of the ENO1-specific Tcc, 51 were CD4+, 21 (41\%) produced IL-17 (Th17) and among these 12 (24\%) secreted both IL-17 and IFN- $\gamma$ (Fig. 1D). A total of 15 ENO1-specific $\mathrm{CD}^{+}{ }^{+}$Tcc secreted IL-4, either alone $(6 \mathrm{Th} 2,12 \%)$ or with IFN- $\gamma\left(9\right.$ Th0, 18\%). Finally, 14 (27\%) ENO1-specific CD4 ${ }^{+}$ Tcc produced only IFN- $\gamma$, showing a Th1 profile. Notably, taken together, of the 14 Th1 clones and the 12 Th17 clones producing IFN $-\gamma$ and the 9 Th0 Tcc, we found that the majority of ENO1-specific CD4 ${ }^{+}$Tcc $(\%)$ were able to produce IFN- $\gamma$. The last ENO1-specific CD4 ${ }^{+}$Tcc was FoxP3 ${ }^{+}$and produced IL-10 and TGF- $\beta$, showing a Treg profile. Of the remaining three ENO1-specific $\mathrm{CD}^{+} \mathrm{Tcc}$, one had a regulatory profile (defined as Tcreg) and two had a Th1 profile (defined as Tc1) (Fig. 1D).

Functional properties of ENO1-specific $T$ cell clones. To evaluate the anticancer properties of the ENO1-specific Tcc isolated from the peripheral blood of PDAC patients, we assessed their cytotoxicity using ENO1-pulsed ${ }^{51} \mathrm{Cr}$-labeled autologous EBV-B cells as targets. At an effector-to-target ratio of 10:1, all $14 \mathrm{Th} 1$, and 7 out of $9 \mathrm{Th} 0$ clones were shown to lyse their targets (Fig. 2A). Among the Th17 population, only the 12 Tcc IL-17/IFN- $\gamma$ double producers were able to kill their target cells.

None of the six Th2 or the ENO1-specific Treg Tcc lysed the EBV-B cells, while only the two ENO1-specific Tc1 CD8 ${ }^{+}$ clones killed the EBV-B cells.

As expected, in the presence of anti-HLA class II blocking mAbs, all the ENO1-specific $\mathrm{CD}^{+}{ }^{+} \mathrm{Tcc}$ were unable to kill the EBV-B cells, and similar results were obtained for the two ENO1-specific Tc1 in the presence of anti-HLA class I blocking mAbs (Fig. 2A).

Effector T cells can kill their targets by Fas-FasL-mediated apoptosis, and therefore, the ability of ENO1-specific Tcc to induce ${ }^{51} \mathrm{Cr}$ release by $\mathrm{Fas}^{+} \mathrm{Pancl}$ cells was evaluated. Upon mitogen activation, $57 \%$ of $\mathrm{CD}^{+}(29 / 51)$ and $66 \%$ of $\mathrm{CD}^{+}$ (2/3) ENO1-specific Tcc induced apoptosis in target cells (Fig. 2B). Among the CD4 ${ }^{+}$Tcc, 14 were Th1, 12 were Th17, and 3 were Th0, but none were Th2 or Treg (Fig. 2B). The role of the Fas-FasL interaction in ${ }^{51} \mathrm{Cr}$ release was confirmed by its inhibition (range, 38.3-57.6\%) using an anti-Fas antibody (data not shown).

Finally, we evaluated the ability of the unique ENO1specific Treg Tcc (Tcc 6) to inhibit the proliferation of ENO1-effector Tcc (Th1 and Th17) obtained from the same patient (Pcp 8). As shown in Fig. 2C, the ENO1-specific Treg clone inhibited proliferation of both ENO1-specific effector Tcc: the Th1 (Tcc 18) and the Th17 (Tcc 24).

TCR analysis of ENO-1 specific Tcc from peripheral blood and tumor areas. As ENO1-specific Tcc was detected in both the peripheral blood and tumor areas in $73 \%$ of the analyzed PDA patients (24), we tested the hypothesis that the same T cell clones were circulating from the periphery to the tumor area and vice versa. We reasoned that if at least one Tcc from the periphery and one Tcc from the tumor area of the same PDAC patient showed the same rearrangement of their TCR, this would provide a proof of concept that the same T lymphocyte was able to recirculate from the tumor to the peripheral blood and vice versa.

Three ENO1-specific Tcc (1Th1, 1 Th17 and 1 Tc1) from the blood and from the central tumor area of the same PDAC patient (Pcp9) were analyzed for TCR $\beta$ and $\gamma$ chain rearrangements by using a classical gene clonality assay (36). Results confirmed the identity of the $\mathrm{CD}^{+} \mathrm{Tcc}$ with a Tcl profile from blood and from tumors (Fig. 3). 

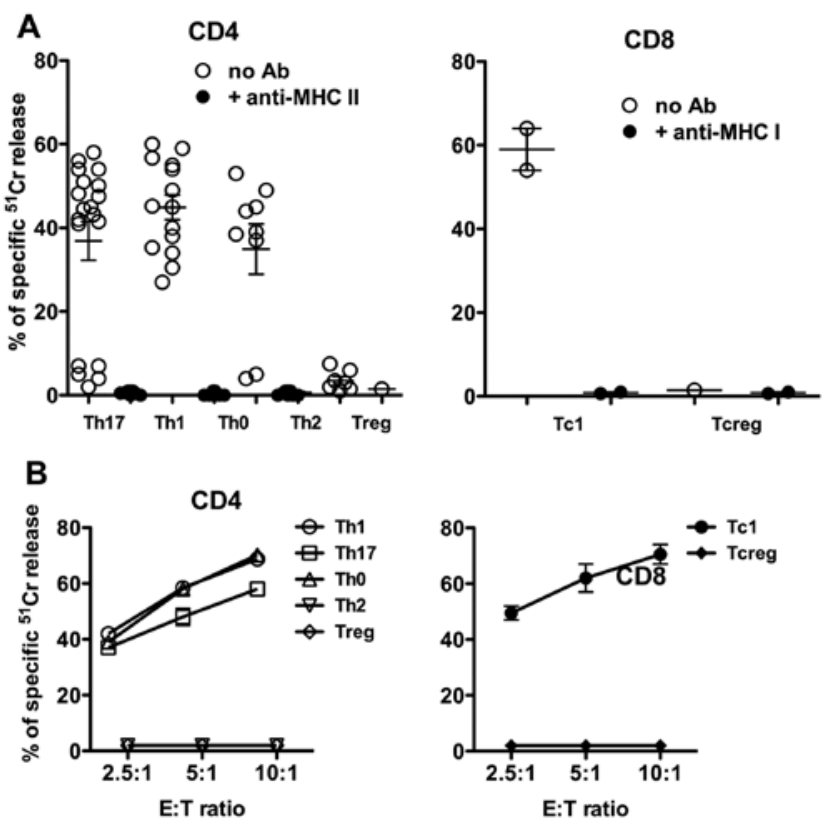

C
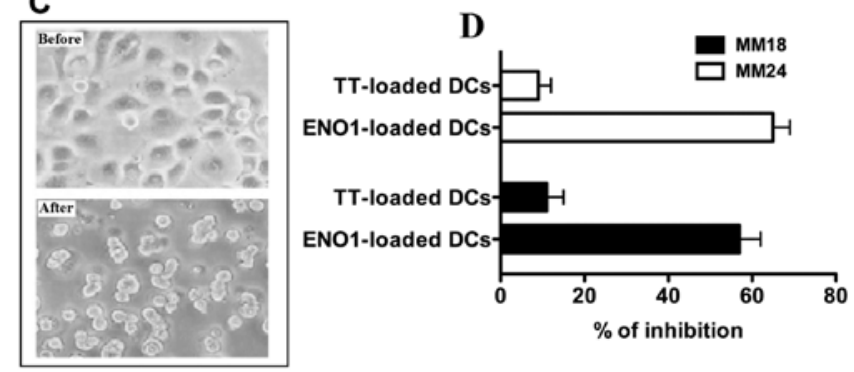

Figure 2. Functional activities of blood-derived Tcc reactive to ENO1. (A) The results are the mean values of percentage-specific ${ }^{51} \mathrm{Cr}$ release at an effector-to-target ratio of 10-to-1 in triplicate micro-cultures. The dotted line represents three SD over the mean ${ }^{51} \mathrm{Cr}$ release in cultures with ${ }^{51} \mathrm{Cr}$-labeled autologous unpulsed EBV-B cells. (B) To assess their ability to induce apoptosis in target cells, ${ }^{51} \mathrm{Cr}$ release was measured as an index of apoptotic target cell death. Data of one representative type clone are reported as mean values of triplicate micro-cultures. (C) Morphological changes in Panc1 cells after co-culture with ENO1-specific Tcc. Typical apoptotic cells with condensed or fragmented nuclei are observed in treated cell lines (bottom panel), while control cells (top panel) show regular-shaped nuclei. All of the photographs are at $\times 200$ magnification. (D) ENO1-specific Treg clones suppresses the proliferation of autologous ENO1-specific effector T cell clones (Th1 and Th17). Results represent mean values and standard deviations of percentage inhibition in triplicate micro-cultures.

Prognostic role of peripheral ENO1-specific T cell clones. Because the majority of ENO1-specific Tcc specifically killed pancreatic cancer cells and were able to re-circulate from the periphery to the tumor and vice versa, we evaluated if the number of peripheral ENO1-specific Tcc correlated with patient survival from diagnosis.

For this purpose, we divided the patients into two groups based on the percentage of peripheral ENO1-specific Tcc and on serum anti-ENO1 specific antibodies: i) $<20$, and ii) $>20$ (Table I). Kaplan-Meier analysis revealed that the patients of group ii showed a better survival compared to group i $(\mathrm{P}<0.0001$, Hazard Ratio=31) suggesting a positive correlation between the percentage of circulating ENO1-specific cells and a longer survival (Fig. 4). In addition, the levels of serum CA19-9 (Table I) in patients of group I were significantly
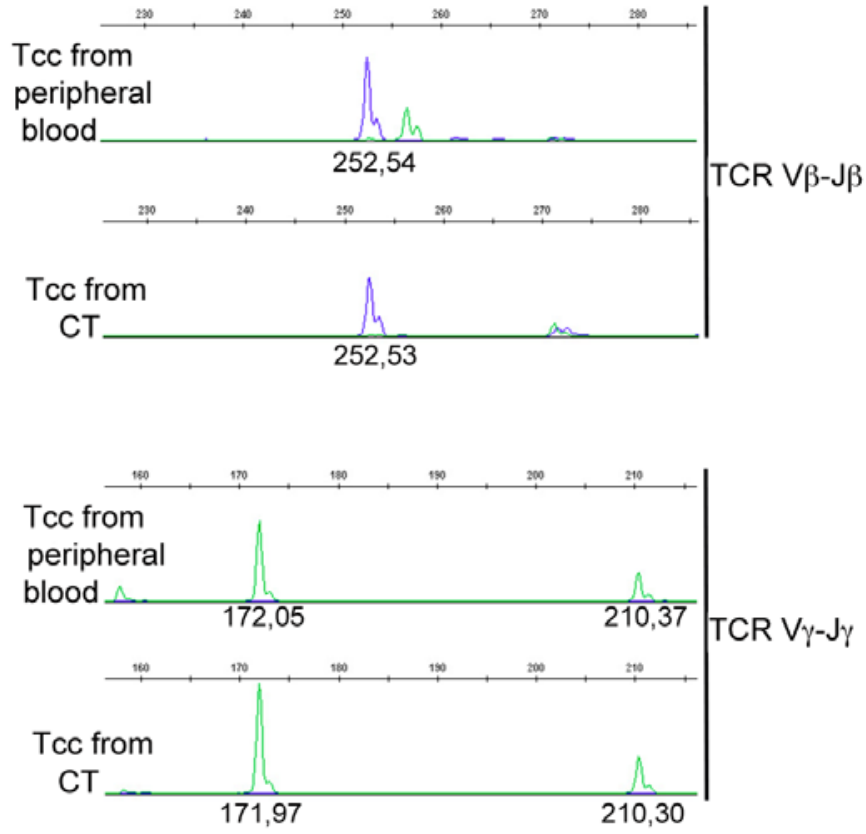

Figure 3. Analysis of TCR gene rearrangement. Molecular analysis of TCR gene rearrangement (BIOMED-2/Euroclonality protocol) of ENO1-specific Tcc isolated from the central tumor area and the peripheral blood of the same PDAC patient. Genescan profiles of TCR $\beta$ (tube A) and TCR $\gamma$ (tube D) are represented. Numbers in square brackets indicate the length of the clonal peaks in base pairs (bp).

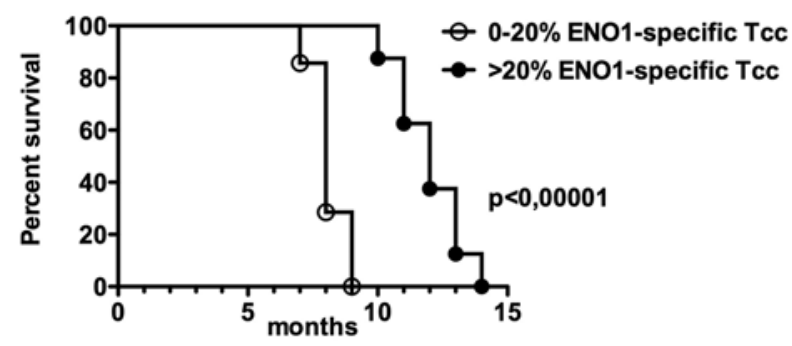

Figure 4. Correlation between patient survival and number of peripheral ENO1-specific Tcc. To correlate the survival of PDAC patients with the number of ENO1-specific Tcc we divided the patients into two groups: i) patients with ENO1-specific Tcc $(\leq 20 \%)$ and ii) patients with ENO1-specific Tcc $(>20 \%)$. We correlated the different groups using a Kaplan-Meier curve. The patients of group ii showed a better survival compared to groups i $(p<0.00001$, Hazard Ratio=31) suggesting a positive correlation between the percentage of circulating ENO1-specific cells and a longer survival.

$(\mathrm{P}<0.0001)$ higher than those in PDAC patients of ii group (data not shown). On the contrary, the PDAC expression of ENO1 is similar in the different patients of the two groups. PDAC tissues diffusely expressed $\alpha$-enolase in the cells lining the neoplastic ducts infiltrating the stroma. In other words, Enolase is overexpressed in most of PC tissues, and to discriminate between high and lower overexpression is difficult. Thus, it is not possible to achieve a correlation between the expression of Enolase and the presence of specific ENO1-specific Tcc.

\section{Discussion}

ENO1 is a PDAC-associated antigen, capable of eliciting an anti-PDAC T cell response in situ, as demonstrated in a previous study, where we characterized the effector functions 


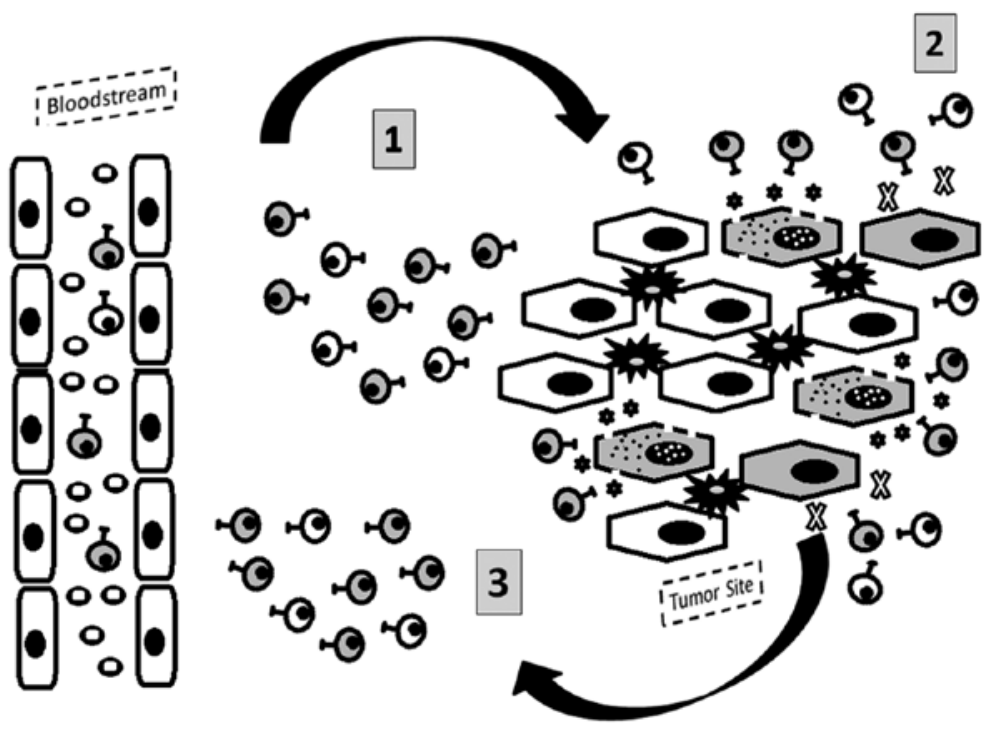

Figure 5. The path of ENO1-specific T cells in pancreatic cancer. Proinflammatory cytokines and chemokines attract T cells specific to ENO1 (and/or to other TAA) from the peripheral blood to the tumor site (1). Under the influence of the tumor microenvironment (which includes stromal cells, extracellular matrix and myeloid suppressor cells), the ENO1-specific T cells undergo different fates: some maintain the effector function (gray) while others shift to regulatory functions (white), which prevent the killing of cancer cells by effector cells and favor cancer cell escape (2). Some of the ENO1-specific Tcc migrate back into the bloodstream, where the number of effector $\mathrm{T}$ cells increases due to the absence of the immunosuppressive action of the tumor microenvironment (3).

of ENO1-specific T-cells isolated from the tumor tissue of PDAC patients (24). We showed that ENO1-specific Th1/Th17 cells have a specific anticancer effector function, but they accumulate in the healthy tissue surrounding the cancer cells, where, conversely, there are elevated levels of ENO1-specific Tregs (24). Based on these observations, we hypothesize that the tumor microenvironment negatively influences the cytolytic activity of $\mathrm{T}$ lymphocytes by promoting the recruitment of specific Treg cells. To investigate this hypothesis, we characterized the ENO1-specific peripheral $\mathrm{T}$ cell response in the same previously-enrolled PDAC patients (24) to ascertain whether or not the $\mathrm{T}$ reg pool was still maintained.

Firstly, we showed that PBMCs from ten PDAC patients were able to proliferate in vitro in the presence of ENO1, while only three healthy controls reacted upon antigen stimulation. This indicates that most PDAC patients have ENO1-specific $\mathrm{T}$ cell precursors in their peripheral blood, which can be triggered by in vitro ENO1 re-stimulation. The presence of the anti ENO1-precursor appears to be PDAC-specific as very few healthy subjects displayed peripheral blood lymphocyte proliferation in response to ENO1 and also, a significantly lower index of proliferation.

To gain information on the functional status of anti-ENO1specific peripheral T lymphocytes, we obtained ENO1-induced $\mathrm{T}$ cell lines and subsequently generated Tcc from all PDAC patients, with the exception of three patients (Pcp 3, 6 and 10). Notably, none of the PBMCs isolated from these three patients proliferated in the presence of ENO1, even though ENO1specific Tcc were obtained from the tumor area in two (Pcp 3 and 10) out of the three patients (24). The lack of ENO1-specific $\mathrm{T}$ cells in the peripheral blood of these patients, is probably due to a difference in the migratory pattern of tumor-specific $\mathrm{T}$ cells (39), or to differences in the tumor microenvironment, in cytokine/chemokine secretion (40) or altered protein expression (such as integrins), which circumscribe the immune response in situ $(41,42)$.

In addition, we did not analyze ENO1 expression in the tumor tissue of our patients, and we therefore cannot exclude the possibility that the absence of a T-cell response in one patient may result from reduced levels of ENO1 expression. We have shown, in fact, that the anti-ENO1 humoral and cellular responses are induced as a consequence of enhanced ENO1 expression in PDAC tissues (24).

However, the most important result is that the peripheral anticancer response is a 'mirror' of the intratumoral PDACspecific response, clearly demonstrated as the majority of patients displayed both immune responses with only two exceptions. Thus, analysis of the Tcc of the peripheral immune response in PDAC may be an adequate method to determine if a patient will have a cancer-specific immune response in situ.

The peripheral blood of PDAC patients has been shown to display serum anti-ENO1 IgG autoantibodies (26) and here, we provide the first evidence that, in PDAC patients, the overall percentage of peripheral ENO1-specific T cells, reaches one third of the total Tcc obtained.

With the exception of one patient (Pcp10), all patients had detectable serum anti-ENO1 IgG, and interestingly, those with higher titers had the highest percentage of ENO1-specific $\mathrm{T}$ clones, in particular patients 9 and 15 .

Remarkably, out of the 54 ENO1-specific Tcc obtained, 51 were $\mathrm{CD}^{+}$and the vast majority of them $(69 \%)$ displayed a Th1/Th17 profile, thus conferring to them a potential anticancer role (43-46). Of the remaining $\mathrm{CD}^{+} \mathrm{Tcc}$, one displayed a Treg profile and two were Tc1.

Previously, we have confirmed the immune-suppressive role of the PDAC microenvironment, demonstrating that intratumoral ENO1-specific CD4 ${ }^{+} \mathrm{T}$ cells displayed an increased number of $\mathrm{T}$ null cells and a Treg phenotype (24). On the 
contrary, the present data demonstrate that, within the same patients, circulating ENO1-specific Tcc showed a different cytokine profile compared to Tcc isolated from the tumoral tissue, with an increased IFN- $\gamma$ secretion and a decreased regulatory profile. In agreement with these findings, we have also demonstrated that the few Tc1 and all the ENO1-specific IFN- $\gamma$-producing CD $4^{+}$Tcc had cytotoxic activity and most of them induced apoptosis in target cells, showing an effective anticancer role.

Of particular relevance, the analysis of TCR rearrangements of some ENO1-specific peripheral and intratumoral Tcc from the same PDAC patient confirmed the ability of tumorinfiltrating lymphocytes to circulate. Therefore, cytotoxic ENO1-specific Tcc that reach the tumor could be subsequently modified and switched off by different immunosuppressive mechanisms including PD-1/PD-L1 $(41,42)$. However, because of the retained cytotoxic ability and potential antitumor function of peripheral ENO1-specific Tcc, they could be very important for the elimination of cancer-circulating cells and prevention of metastasis and disease relapse (47,48) (Fig. 5).

Finally, we emphasize that patients with a high number of peripheral blood ENO1-specific Tcc showed a significant improved survival compared to PDAC patients without or with a low number of ENO1-specific Tcc. However, we are aware that only a small cohort was employed in the present study, and while the presence of Tcc could be considered a potential prognostic marker for PDAC, it should be validated in a larger cohort of PDAC patients. These results, therefore, indicate the relevance of the ENO1-specific immune response in the generation of an effective anticancer response, which correlates with improved survival of PDAC patients.

In conclusion, we have demonstrated for the first time that, in PDAC patients: i) there was a correspondence between the intratumoral and peripheral ENO1-specific immune response; ii) the same clonal $\mathrm{T}$ cells recirculated from the tumor to the periphery; and iii) the circulating ENO1-specific T cells showed a more effective anticancer role compared to Tcc isolated from the tumoral tissue.

These data support the idea that ENO1 could be a good candidate antigen for immunotherapeutic treatments in PDAC patients (49), in particular after surgical resection to prevent relapse of the disease through the marked anticancer activity of circulating ENO1-specific Tcc. Additional studies should be carried out to identify the different immunosuppressive mechanisms that are able to modify or switch off the anticancer specific immune response in order to make this immunotherapeutic approach feasible.

\section{Acknowledgements}

The present study was supported by grants of the Italian Ministry of University and Research (PRIN 2009), the University of Florence, the Associazione Italiana Ricerca sul Cancro (AIRC IG no. 11643 and 5x1000 no. 12182), the University of Torino-Progetti di Ateneo 2011 (grant RetheORTO11RKTW), the Seventh Framework Program of European Community (European Pancreatic Cancer-Tumor Microenvironment-Network, EPC-TM-Net, no. 256974), the Fondazione Internazionale di Medicina Sperimentale, and partly by the FAS-funded 'Programma Attuativo Regionale
(Toscana)'. We thank Dr Radhika Srinivasan for English revision of the manuscript.

\section{References}

1. Hartwig W, Werner J, Jäger D, Debus J and Büchler MW: Improvement of surgical results for pancreatic cancer. Lancet Oncol 14: e476-e485, 2013.

2. Wolfgang CL, Herman JM, Laheru DA, Klein AP, Erdek MA, Fishman EK and Hruban RH: Recent progress in pancreatic cancer. CA Cancer J Clin 63: 318-348, 2013.

3. Singh P, Srinivasan R and Wig JD: Major molecular markers in pancreatic ductal adenocarcinoma and their roles in screening, diagnosis, prognosis, and treatment. Pancreas 40: 644-652, 2011.

4. Loos M, Giese NA, Kleeff J, Giese T, Gaida MM, Bergmann F, Laschinger M, W Büchler M and Friess $\mathrm{H}$ : Clinical significance and regulation of the costimulatory molecule $\mathrm{B} 7-\mathrm{H} 1$ in pancreatic cancer. Cancer Lett 268: 98-109, 2008.

5. Bazhin AV, Shevchenko I, Umansky V, Werner J and Karakhanova S: Two immune faces of pancreatic adenocarcinoma: Possible implication for immunotherapy. Cancer Immunol Immunother 63: 59-65, 2014.

6. Galon J, Costes A, Sanchez-Cabo F, Kirilovsky A, Mlecnik B, Lagorce-Pagès $\mathrm{C}$, Tosolini $\mathrm{M}$, Camus $\mathrm{M}$, Berger $\mathrm{A}$, Wind $\mathrm{P}$, et al: Type, density, and location of immune cells within human colorectal tumors predict clinical outcome. Science 313: 1960-1964, 2006.

7. Ryschich E, Nötzel T, Hinz U, Autschbach F, Ferguson J, Simon I, Weitz J, Fröhlich B, Klar E, Büchler MW, et al: Control of T-cellmediated immune response by HLA class I in human pancreatic carcinoma. Clin Cancer Res 11: 498-504, 2005.

8. Nomi T, Sho M, Akahori T, Hamada K, Kubo A, Kanehiro H, Nakamura S, Enomoto K, Yagita H, Azuma M, et al: Clinical significance and therapeutic potential of the programmed death-1 ligand/programmed death-1 pathway in human pancreatic cancer. Clin Cancer Res 13: 2151-2157, 2007.

9. Park IH, Kong SY, Ro JY, Kwon Y, Kang JH, Mo HJ, Jung SY, Lee S, Lee KS, Kang HS, et al: Prognostic implications of tumorinfiltrating lymphocytes in association with programmed death ligand 1 expression in early-stage breast cancer. Clin Breast Cancer 16: 51-58, 2015.

10. Blank C, Gajewski TF and Mackensen A: Interaction of PD-L1 on tumor cells with PD-1 on tumor-specific T cells as a mechanism of immune evasion: Implications for tumor immunotherapy. Cancer Immunol Immunother 54: 307-314, 2005.

11. Chen Y, Sun J, Zhao H, Zhu D, Zhi Q, Song S, Zhang L, He S, Kuang Y, Zhang Z, et al: The coexpression and clinical significance of costimulatory molecules B7-H1, B7-H3, and B7-H4 in human pancreatic cancer. Onco Targets Ther 7: 1465-1472, 2014.

12. Wadle A, Kubuschok B, Imig J, Wuellner B, Wittig C, Zwick C, Mischo A, Waetzig K, Romeike BF, Lindemann W, et al: Serological immune response to cancer testis antigens in patients with pancreatic cancer. Int J Cancer 119: 117-125, 2006.

13. Schmitz-Winnenthal FH, Galindo-Escobedo LV, Rimoldi D, Geng W, Romero P, Koch M, Weitz J, Krempien R, Niethammer AG, Beckhove P, et al: Potential target antigens for immunotherapy in human pancreatic cancer. Cancer Lett 252: 290-298, 2007.

14. Gnjatic S, Ritter E, Büchler MW, Giese NA, Brors B, Frei C, Murray A, Halama N, Zörnig I, Chen YT, et al: Seromic profiling of ovarian and pancreatic cancer. Proc Natl Acad Sci USA 107: 5088-5093, 2010.

15. Tomaino B, Cappello P, Capello M, Fredolini C, Ponzetto A, Novarino A, Ciuffreda L, Bertetto O, De Angelis C, Gaia E, et al: Autoantibody signature in human ductal pancreatic adenocarcinoma. J Proteome Res 6: 4025-4031, 2007.

16. Kubuschok B, Neumann F, Breit R, Sester M, Schormann C, Wagner C, Sester U, Hartmann F, Wagner M, Remberger K, et al: Naturally occurring T-cell response against mutated p21 ras oncoprotein in pancreatic cancer. Clin Cancer Res 12: 1365-1372, 2006.

17. Wenandy L, Sørensen RB, Sengeløv L, Svane IM, thor Straten P and Andersen MH: The immunogenicity of the hTERT540-548 peptide in cancer. Clin Cancer Res 14: 4-7, 2008. 
18. Gjertsen MK, Bakka A, Breivik J, Saeterdal I, Solheim BG, Søreide O, Thorsby E and Gaudernack G: Vaccination with mutant ras peptides and induction of T-cell responsiveness in pancreatic carcinoma patients carrying the corresponding RAS mutation. Lancet 346: 1399-1400, 1995.

19. Wobser M, Keikavoussi P, Kunzmann V, Weininger M, Andersen $\mathrm{MH}$ and Becker JC: Complete remission of liver metastasis of pancreatic cancer under vaccination with a HLA-A 2 restricted peptide derived from the universal tumor antigen survivin. Cancer Immunol Immunother 55: 1294-1298, 2006.

20. Yamaguchi K, Enjoji M and Tsuneyoshi M: Pancreatoduodenal carcinoma: A clinicopathologic study of 304 patients and immunohistochemical observation for CEA and CA19-9. J Surg Oncol 47: 148-154, 1991.

21. Komoto M, Nakata B, Amano R, Yamada N, Yashiro M, Ohira M, Wakasa K and Hirakawa K: HER2 overexpression correlates with survival after curative resection of pancreatic cancer. Cancer Sci 100: 1243-1247, 2009.

22. Heller A, Zörnig I, Müller T, Giorgadze K, Frei C, Giese T, Bergmann F, Schmidt J, Werner J, Buchler MW, et al: Immunogenicity of SEREX-identified antigens and disease outcome in pancreatic cancer. Cancer Immunol Immunother 59: $1389-1400,2010$

23. Cappello P, Tomaino B, Chiarle R, Ceruti P, Novarino A, Castagnoli C, Migliorini P, Perconti G, Giallongo A, Milella M, et al: An integrated humoral and cellular response is elicited in pancreatic cancer by alpha-enolase, a novel pancreatic ductal adenocarcinoma-associated antigen. Int J Cancer 125: 639-648, 2009.

24. Amedei A, Niccolai E, Benagiano M, Della Bella C, Cianchi F, Bechi P, Taddei A, Bencini L, Farsi M, Cappello P, et al: Ex vivo analysis of pancreatic cancer-infiltrating T lymphocytes reveals that ENO-specific Tregs accumulate in tumor tissue and inhibit Th1/Th17 effector cell functions. Cancer Immunol Immunother 62: 1249-1260, 2013.

25. Capello M, Ferri-Borgogno S, Cappello P and Novelli F: $\alpha$-Enolase: A promising therapeutic and diagnostic tumor target. FEBS J 278: 1064-1074, 2011.

26. Tomaino B, Cappello P, Capello M, Fredolini C, Sperduti I, Migliorini P, Salacone P, Novarino A, Giacobino A, Ciuffreda L, et al: Circulating autoantibodies to phosphorylated $\alpha$-enolase are a hallmark of pancreatic cancer. J Proteome Res 10: 105-112, 2011.

27. Cappello P, Rolla S, Chiarle R, Principe M, Cavallo F, Perconti G, Feo S, Giovarelli M and Novelli F: Vaccination with ENO1 DNA prolongs survival of genetically engineered mice with pancreatic cancer. Gastroenterology 144: 1098-1106, 2013.

28. De Monte L, Reni M, Tassi E, Clavenna D, Papa I, Recalde H, Braga M, Di Carlo V, Doglioni C and Protti MP: Intratumor T helper type 2 cell infiltrate correlates with cancer-associated fibroblast thymic stromal lymphopoietin production and reduced survival in pancreatic cancer. J Exp Med 208: 469-478, 2011.

29. Linehan DC and Goedegebuure PS: CD $25^{+} \mathrm{CD}^{+}$regulatory T-cells in cancer. Immunol Res 32: 155-168, 2005.

30. Liyanage UK, Moore TT, Joo HG, Tanaka Y, Herrmann V, Doherty G, Drebin JA, Strasberg SM, Eberlein TJ, Goedegebuure PS, et al: Prevalence of regulatory T cells is increased in peripheral blood and tumor microenvironment of patients with pancreas or breast adenocarcinoma. J Immunol 169: 2756-2761, 2002

31. Bettelli E, Oukka M and Kuchroo VK: T(H)-17 cells in the circle of immunity and autoimmunity. Nat Immunol 8: 345-350, 2007.

32. Kryczek I, Wei S, Zou L, Altuwaijri S, Szeliga W, Kolls J, Chang A and Zou W: Cutting edge: Th17 and regulatory T cell dynamics and the regulation by IL-2 in the tumor microenvironment. J Immunol 178: 6730-6733, 2007.
33. Zhang B, Rong G, Wei H, Zhang M, Bi J, Ma L, Xue X, Wei G, Liu X and Fang G: The prevalence of Th17 cells in patients with gastric cancer. Biochem Biophys Res Commun 374: 533-537, 2008.

34. Koyama K, Kagamu H, Miura S, Hiura T, Miyabayashi T, Itoh R, Kuriyama H, Tanaka H, Tanaka J, Yoshizawa H, et al: Reciprocal CD4 $4^{+}$T-cell balance of effector CD62Llow CD $4^{+}$and $\mathrm{CD} 62 \mathrm{LhighCD} 25^{+} \mathrm{CD} 4^{+}$regulatory $\mathrm{T}$ cells in small cell lung cancer reflects disease stage. Clin Cancer Res 14: 6770-6779, 2008.

35. Warshaw AL and Fernández-del Castillo C: Pancreatic carcinoma. N Engl J Med 326: 455-465, 1992.

36. Amedei A, Della Bella C, Niccolai E, Stanflin N, Benagiano M, Duranti R, Del Prete G, Murphy TF and D'Elios MM: Moraxella catarrhalis-specific Th1 cells in BAL fluids of chronic obstructive pulmonary disease patients. Int J Immunopathol Pharmacol 22: 979-990, 2009.

37. Amedei A, Niccolai E, Della Bella C, Cianchi F, Trallori G, Benagiano M, Bencini L, Bernini M, Farsi M, Moretti R, et al: Characterization of tumor antigen peptide-specific $\mathrm{T}$ cells isolated from the neoplastic tissue of patients with gastric adenocarcinoma. Cancer Immunol Immunother 58: 1819-1830, 2009.

38. van Dongen JJ, Langerak AW, Brüggemann M, Evans PA, Hummel M, Lavender FL, Delabesse E, Davi F, Schuuring E, García-Sanz R, et al: Design and standardization of PCR primers and protocols for detection of clonal immunoglobulin and T-cell receptor gene recombinations in suspect lymphoproliferations: Report of the BIOMED-2 Concerted Action BMH4-CT98-3936. Leukemia 17: 2257-2317, 2003

39. Nagorsen D, Scheibenbogen C, Schaller G, Leigh B, Schmittel A, Letsch A, Thiel E and Keilholz U: Differences in T-cell immunity toward tumor-associated antigens in colorectal cancer and breast cancer patients. Int J Cancer 105: 221-225, 2003.

40. Lippitz BE: Cytokine patterns in patients with cancer: A systematic review. Lancet Oncol 14: e218-e228, 2013.

41. Berlin C, Berg EL, Briskin MJ, Andrew DP, Kilshaw PJ, Holzmann B, Weissman IL, Hamann A and Butcher EC: Alpha 4 beta 7 integrin mediates lymphocyte binding to the mucosal vascular addressin MAdCAM-1. Cell 74: 185-195, 1993.

42. Rott LS, Briskin MJ, Andrew DP, Berg EL and Butcher EC: A fundamental subdivision of circulating lymphocytes defined by adhesion to mucosal addressin cell adhesion molecule-1. Comparison with vascular cell adhesion molecule-1 and correlation with beta 7 integrins and memory differentiation. J Immunol 156: 3727-3736, 1996.

43. Brandacher G, Winkler C, Schroecksnadel K, Margreiter R and Fuchs D: Antitumoral activity of interferon-gamma involved in impaired immune function in cancer patients. Curr Drug Metab 7: 599-612, 2006.

44. Zaidi MR and Merlino G: The two faces of interferon- $\gamma$ in cancer. Clin Cancer Res 17: 6118-6124, 2011.

45. Neurath MF and Finotto S: The emerging role of T cell cytokines in non-small cell lung cancer. Cytokine Growth Factor Rev 23: 315-322, 2012.

46. Hemdan NY: Anti-cancer versus cancer-promoting effects of the interleukin-17-producing T helper cells. Immunol Lett 149: 123-133, 2013.

47. Bidard FC, Huguet F, Louvet C, Mineur L, Bouché O, Chibaudel B, Artru P, Desseigne F, Bachet JB, Mathiot C, et al: Circulating tumor cells in locally advanced pancreatic adenocarcinoma: The ancillary CirCe 07 study to the LAP 07 trial. Ann Oncol 24: 2057-2061,2013.

48. Tjensvoll K, Nordgård $\mathrm{O}$ and Smaaland R: Circulating tumor cells in pancreatic cancer patients: Methods of detection and clinical implications. Int J Cancer 134: 1-8, 2014.

49. Niccolai E, Prisco D, D’Elios MM and Amedei A: What is recent in pancreatic cancer immunotherapy? BioMed Res Int 2013 492372, 2013. 